\section{Pacific Northwest}

National Laboratory

Operated by Battelle for the

U.S. Department of Energy

\title{
Provenance Store Evaluation
}

PR Paulson

TD Gibson

KL Schuchardt

EG Stephan

March 2008

Prepared for the U.S. Department of Energy under Contract DE-AC05-76RL01830

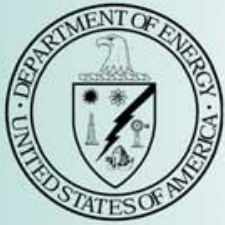




\section{DISCLAIMER}

This report was prepared as an account of work sponsored by an agency of the United States Government. Neither the United States Government nor any agency thereof, nor Battelle Memorial Institute, nor any of their employees, makes any warranty, express or implied, or assumes any legal liability or responsibility for the accuracy, completeness, or usefulness of any information, apparatus, product, or process disclosed, or represents that its use would not infringe privately owned rights. Reference herein to any specific commercial product, process, or service by trade name, trademark, manufacturer, or otherwise does not necessarily constitute or imply its endorsement, recommendation, or favoring by the United States Government or any agency thereof, or Battelle Memorial Institute. The views and opinions of authors expressed herein do not necessarily state or reflect those of the United States Government or any agency thereof.

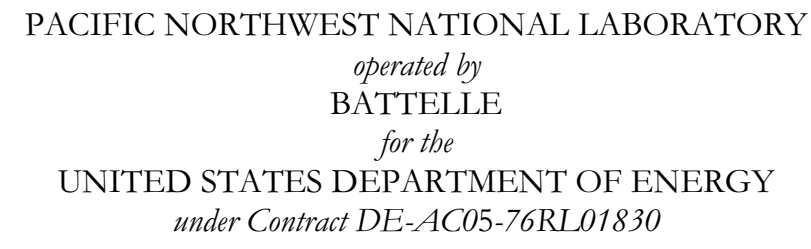

Printed in the United States of America Available to DOE and DOE contractors from the Office of Scientific and Technical Information, P.O. Box 62, Oak Ridge, TN 37831-0062; ph: (865) 576-8401 fax: (865) $576-5728$

email: reports@adonis.osti.gov

Available to the public from the National Technical Information Service, U.S. Department of Commerce, 5285 Port Royal Rd., Springfield, VA 22161 ph: (800) 553-6847 fax: (703) $605-6900$

email: orders@ntis.fedworld.gov online ordering: http://www.ntis.gov/ordering.htm 
PNNL-17237

\section{Provenance Store Evaluation}

P Paulson

T Gibson

K Schuchardt

E Stephan

March 2008

Prepared for

the U.S. Department of Energy

under Contract DE-AC05-76RL01830

Pacific Northwest National Laboratory

Richland, Washington 99352 


\section{Summary}

Requirements for the provenance store and access API are developed. Existing RDF stores and APIs are evaluated against the requirements and performance benchmarks. The team's conclusion is to use MySQL as a database backend, with a possible move to Oracle in the near-term future. Both Jena and Sesame's APIs will be supported, but new code will use the Jena API. 



\section{Contents}

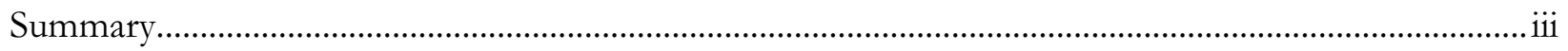

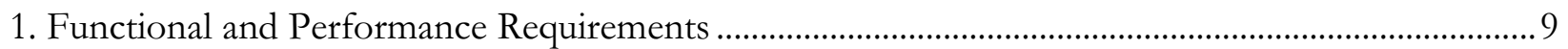

1.1. Provenance creation, recording, and querying ....................................................................... 9

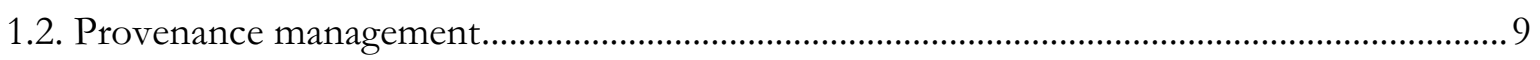

1.2.1 Data security, Reliability, Availability, and Fault-toleranance .................................. 9

1.2.2 Capacity, Scalability, and Extensibility …................................................................ 9

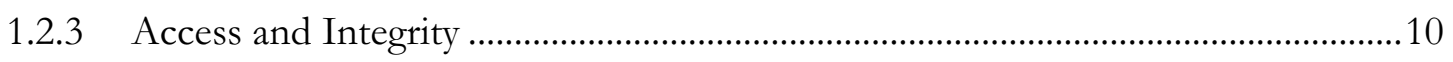

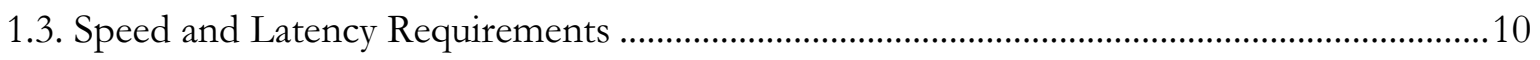

2. Evaluation of RDF Stores for Provenance Recording .................................................................... 11

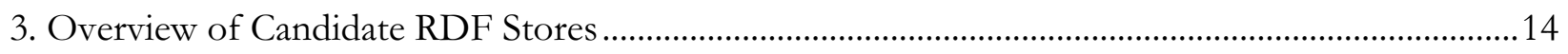

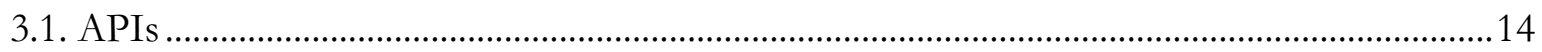

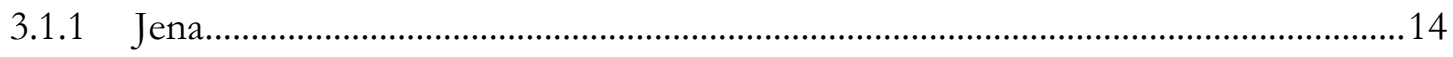

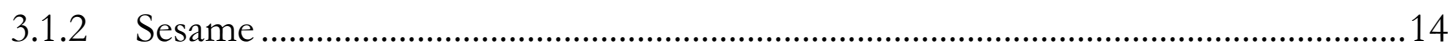

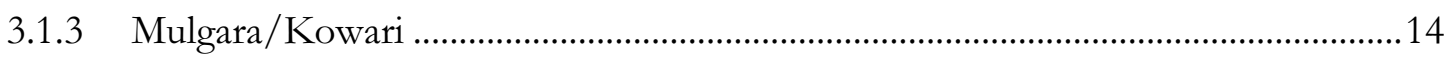

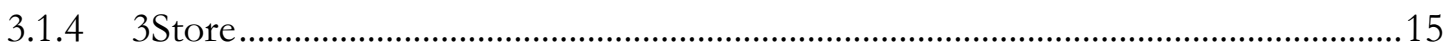

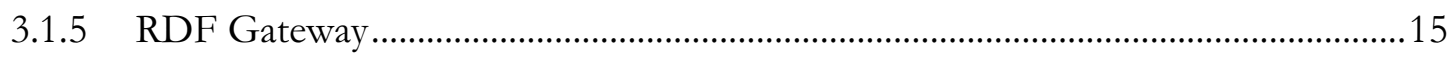

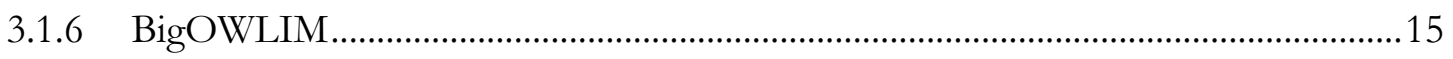

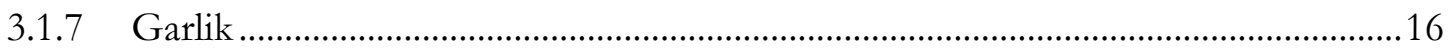

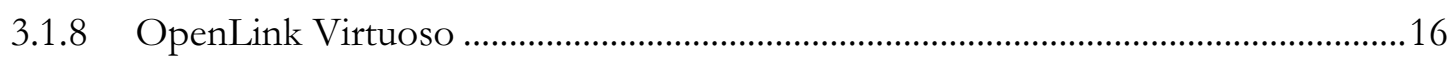

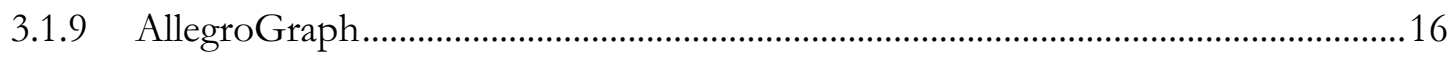

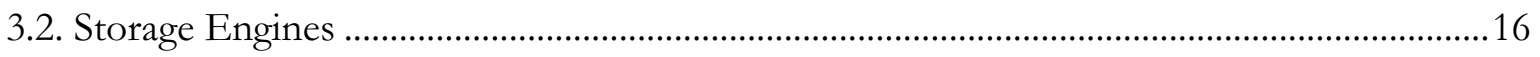

3.2.1 Full feature SQL-based Relational systems ...........................................................16

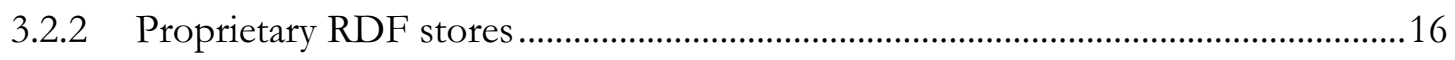

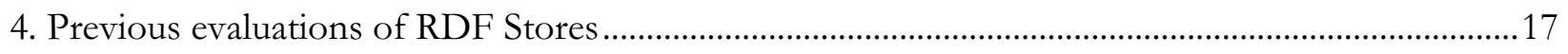

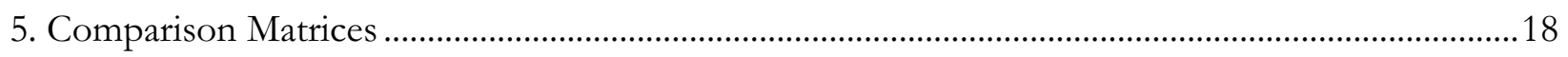

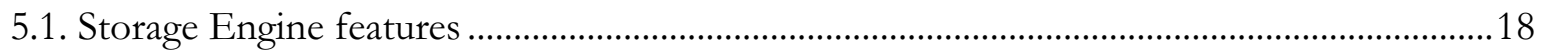

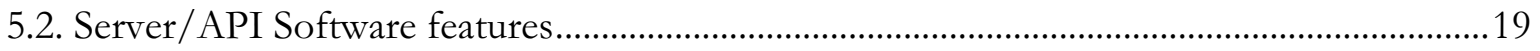

5.3. API/Backend Compatibility …….......................................................................................... 19

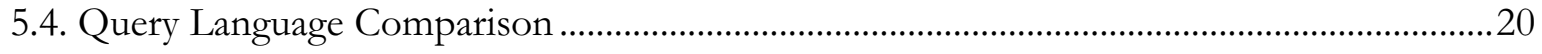

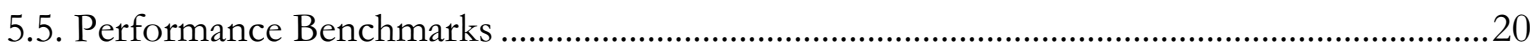

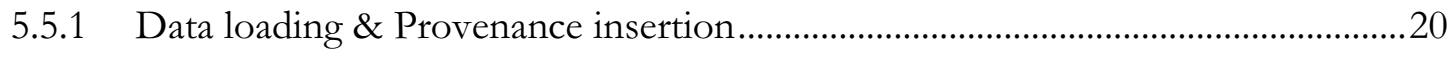

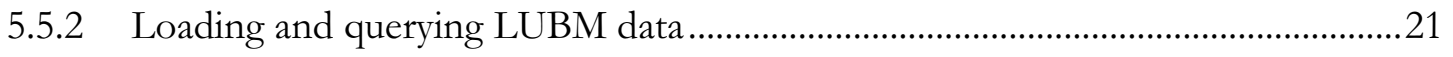




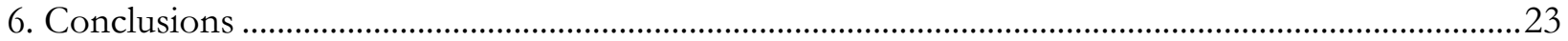

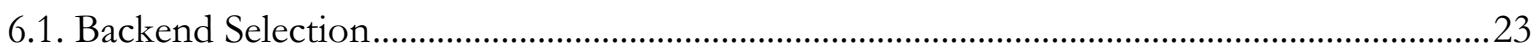

6.2. API Selection …………………………………………………………………………… 23

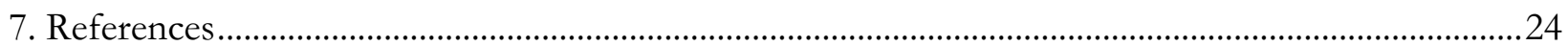




\section{Figures}

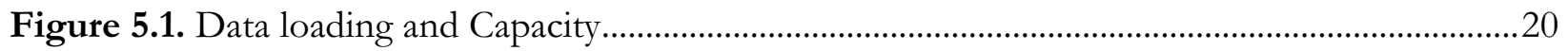




\section{Tables}

Table 2.1. Criteria applied to multiple system components ..............................................................12

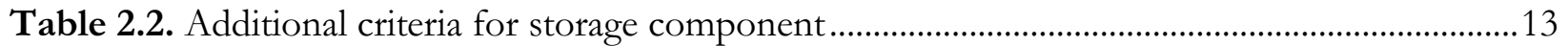

Table 5.1. Comparison of Storage Engine Features .............................................................................18

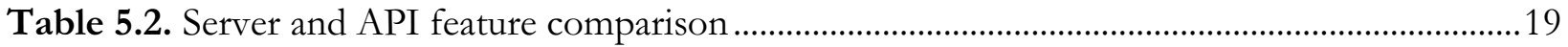

Table 5.3. Compatability between backends and APIs.......................................................................19

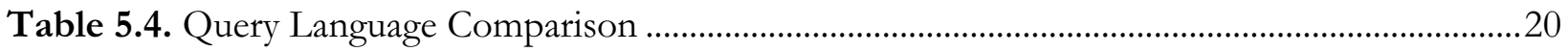

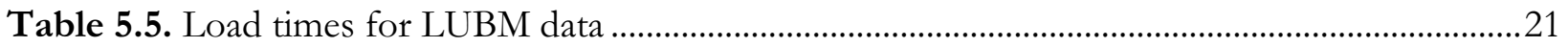

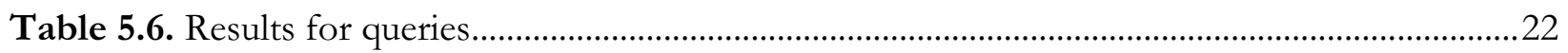




\section{Functional and Performance Requirements}

We want to support the provenance steps described by (Groth, Miles et al. 2006). (Munroe, Groth et al. 2006) specify 4 phases in the provenance lifecycle: creation, recording, querying, and managing. The RDF based Provenance store should support each of the phases.

\subsection{Provenance creation, recording, and querying}

The provenance store should provide APIs or web services to allow users to specify new provenance information; it must also support the storage of a large amount of provenance information. In addition, the store must support queries for all provenance related to some data instance. This may require substantial time to transitively find all information related to a data item.

\subsection{Provenance management}

The system needs to provide tools to support standard data-management tasks. These tasks may include backups and restore, journaling and crash-recovery, purging, datareorganization, and storage optimization.

\subsubsection{Data security, Reliability, Availability, and Fault-toleranance}

Because the projected customers require global access, the system should be capable of $24 \mathrm{X} 7$ operations, which requires online data backup and recovery. Failure of the provenance store should not prevent the execution of client processes; ideally, local provenance stores can provide temporary storage in case of network or server failure. Fail-over processing should be provided.

\subsubsection{Capacity, Scalability, and Extensibility}

Provenance assertions will be generated for every intermediate result generated by the system. We're assuming that the result sets will have high granularity — that is, there will not be provenance associated with each item in a dataset, but the data set as a whole. Historical provenance records will be kept for a window, but a purge process can be created to remove records which are unused.

We're assuming this implies that the capacity must be at least on the order of millions of data-items. Potentially, the system should be able to scale to the order or trillions of dataitems. 


\subsubsection{Access and Integrity}

It is assumed that access to actual data-items will be controlled by client systems. Although not envisioned for prototype systems, user-level access control should be supported for provenance records.

The system should support ACID Transaction support and journaling. Once a client receives confirmation of a commit, all p-assertions submitted as a transaction are guaranteed to persist in the store; if confirmation of a commit is not sent, the persisted store will not reflect any of the processing steps taken as part of the transaction.

\subsection{Speed and Latency Requirements}

For provenance creation, recording, and querying, the system should not cause significant delays to client programs; as much as possible, any additional processing time should be deterministic. 


\section{Evaluation of RDF Stores for Provenance Recording}

Using the requirements as a guideline, we can come up with a set of dimensions that can be used in evaluation of potential RDF Storage systems. The RDF stores under consideration are composed of several components, some of which are interoperable between systems. A preliminary decomposition identifies 3 system components-the storage engine (such as MySQL tables or proprietary file system), API (such as Jena or OpenRdf), and the server software (Joseki is one example). Many of the dimensions described below apply to only 1 component. In addition, some capabilities apply only to the query languages the system's API and server software support. Table 2.1 outlines criteria to evaluate system components. Table 2.2 gives criteria that are only applicable to the server component. 


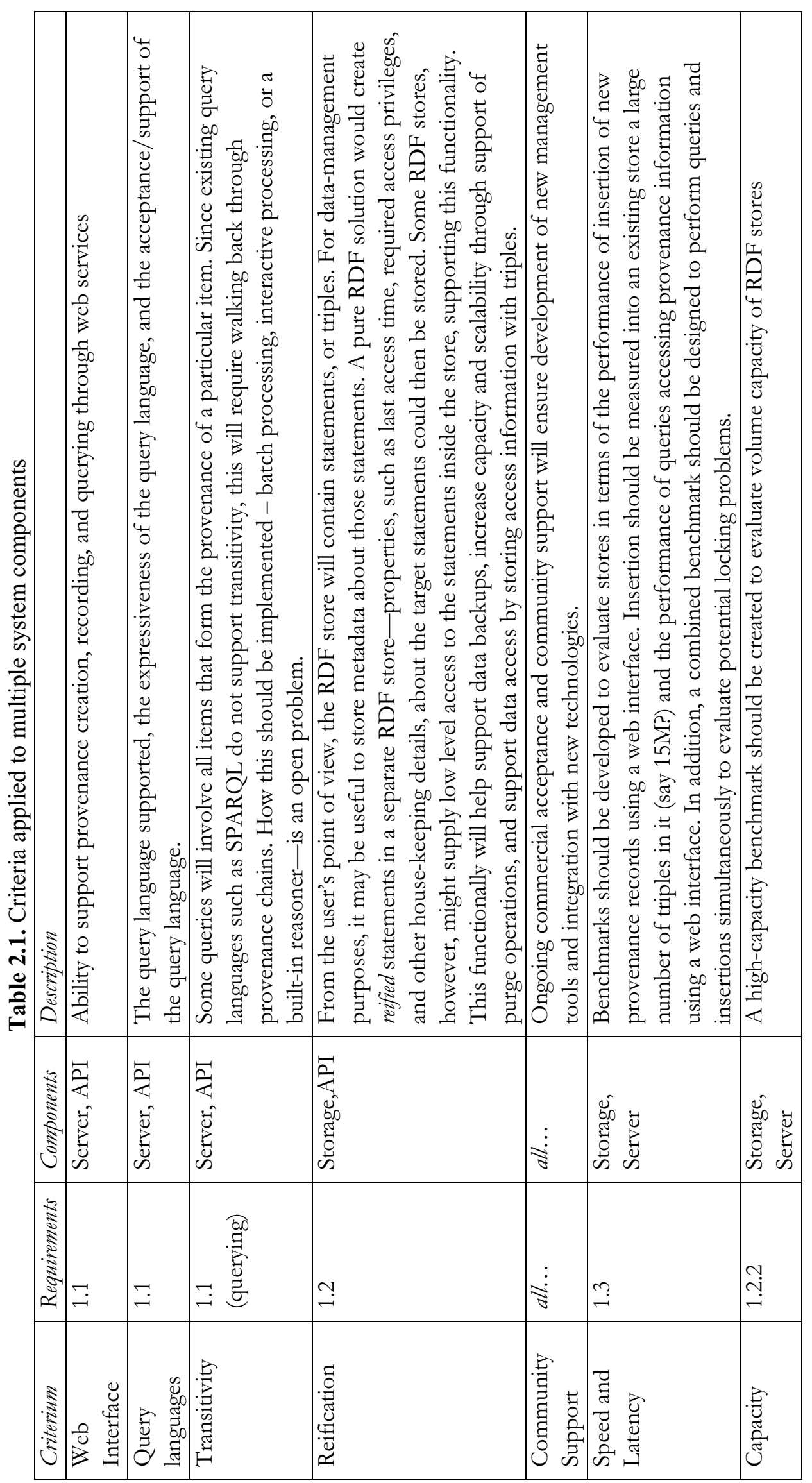




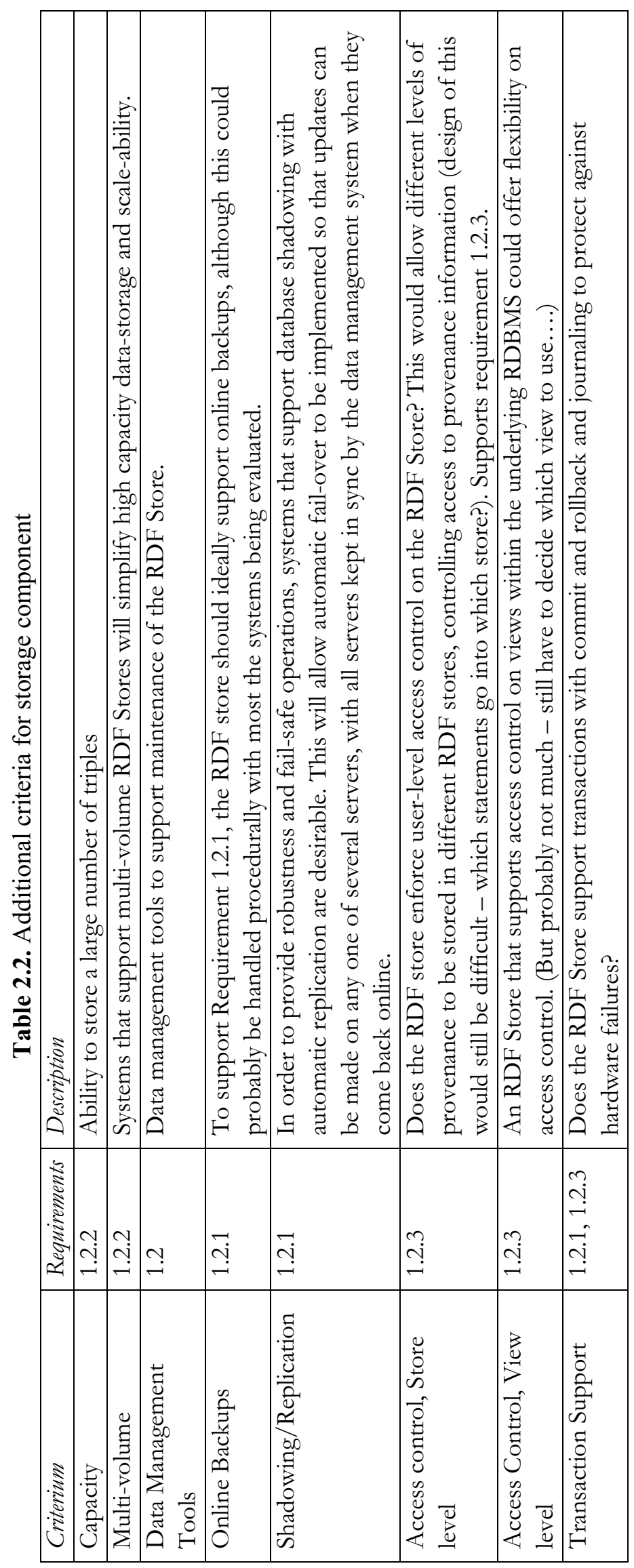




\section{Overview of Candidate RDF Stores}

\subsection{APIs}

\subsubsection{Jena}

Jena (http://jena.sourceforge.net/) provides

1. An API for manipulating RDF graphs

2. Support for multiple reasoning engines - OWL-DL (through Pellet), OWL-Lite, and RDF Schema

3. Support for multiple back-end storage systems, including

a. native support for in-memory graphs

b. RDBMS table storage, implemented for Oracle, SQL Server, MySQL, and Postgres

4. Support for the SPARQL query language

5. Server software (Joseki) that supports the SPARQL query language

Web sources (http://esw.w3.org/topic/Large'TripleStores) indicate installations handling $200 \mathrm{M}$ triples using Postgres as the storage engine.

\subsubsection{Sesame}

Sesame provides

1. An API for manipulating RDF graphs

2. Server software that supports the SeRQL query language

3. Support for a proprietary, file-based storage system

4. Reasoning over RDF Schema

Version 1.0 of Sesame also supported RDBMS table-based storage, but this has not yet been implemented for version 2.0. I was unable to decipher the documentation for version 1.0 support. Web sources indicate fair performance with systems of up to $70 \mathrm{M}$ triples.

\subsubsection{Mulgara/Kowari}

Mulgara is an open-source fork of Kowari. The marketing literature indicates that the design is meant to be scaleable to extremely large graphs. The system uses memory-mapped files and is tailored to 64-bit systems. Web sources indicates good performance with stores of 160M triples (http://esw.w3.org/topic/Large'TripleStores)

Mulgara provides:

1. A server supporting the Itql query/update language

2. A proprietary storage backend 


\subsubsection{Store}

Web sources (http://esw.w3.org/topic/Large'TripleStores) indicate successful applications handling $100 \mathrm{M}$ triples. This product provides a $\mathrm{C}$ language library. Untested since compiling on cygwin didn't go very smoothly - probably best on a Unix or Macintosh, but we're currently benchmarking on a windows machine. Uses MySql as backend.

Provides

1. Sparql Support

2. Store-level access control

3. Uses MySQL

\subsubsection{RDF Gateway}

Web sources(http://esw.w3.org/topic/Large'TripleStores) indicate installations handling 262M triples.

1. Commercial, free for evaluation.

2. RDF Gateway is a complete application and web server that manages a built-in RDF Store.

3. A server supporting the proprietary RDFQL query language. It looks like SPARQL is also supported

4. A proprietary storage backend

5. Access control using NT user and groups

6. Transaction Support

7. 'context' for statement could possibly support statement reification

8. content-level access control

We were unable to determine if on-line backups are supported.

Documentation for this product was too incomplete to allow me to easily code benchmarks for it, although it appears feasible.

\subsubsection{BigOWLIM}

One source claimed that this system handled $1.06 \mathrm{~B}$ statements - adding more statements through OWL inferencing, with a load time was approximately $70 \mathrm{hrs}$.

(http://esw.w3.org/topic/Large'TripleStores).

BigOWLIM is a reasoning and persistence implementation for the Sesame framework. It uses a proprietary disk storage system and implements RDFS and limited OWL entailment (does not support OWL-Lite).

BigOWLIM is not open source- - it was not tested due to licensing limitations. 


\subsubsection{Garlik}

Handles 1.7B triples, according to http://esw.w3.org/topic/Large'TripleStores. www.garlik.com describes a data-privacy monitoring company, very little information is given about their technology. The RDF Store is apparently named JXT, but I found no more information about it using Google.

\subsubsection{OpenLink Virtuoso}

http://esw.w3.org/topic/Large'TripleStores indicates this store handles over 1B triples. This looks like a nice commercial product. Evaluation kits are available for 15 days-not evaluated because we have no license. Supports Sparql.

\subsubsection{AllegroGraph}

Web sources and company information indicate AllegroGraph can handle billions of triples (http://esw.w3.org/topic/Large'TripleStores).

AllegroGraph Allegro graph is single threaded server based rdf store. Multi-volume support

AllegroGraph stores a triple store within a single directory (http://www.franz.com/products/allegrograph/doc/lisp/reference-guide.html).

\subsection{Storage Engines}

\subsubsection{Full feature SQL-based Relational systems}

These systems provide scaleability, multi-volumen support, transaction support, and data management tools. The systems include MySQL, Postgress, Oracle, and SQL server.

\subsubsection{Proprietary RDF stores}

Most of these systems offer little documentation that details the support given for datamanagement tasks, multi-volume support, and transaction support. Proprietary stores include AllegroGraph, the Sesame Native Store, and Mulgara. 


\section{Previous evaluations of RDF Stores}

(Lee 2004) reviews several triple stores, including Jena, Kowari, 3Store, and Sesame. The triple stores were tested in their performance for three specific application tasks'configure', 'display', and 'browse'. In all 3 tasks, when accessing a $21 \mathrm{M}$ triple dataset over a network connection, Sesame performed significantly better than the other contenders.

(Portwin and Parvatikar 2006) examined several RDF stores and chose Jena using Postgres for several reasons, including the existence of proven data-management tools. They found that neither Mulgara nor Sesame was as reliable and scaleable as Jena. They found that while Jena's RDF store was scaleable, its reasoner was not, and that further design decisions were needed to determine how to best support certain types of reasoning. It was also found that Joseki queries required reformulating for optimal results - logically equivelant queries could have a tremendous difference in response times. (Note that this is also true of SQL queries against an RDBMS store, though more kinks have probably been worked out over the years)

TripCom (Triple Space Communication 2006) provides a good overview of the available RDF stores and their characteristics, but does not report any peformance results. 


\section{Comparison Matrices}

\subsection{Storage Engine features}

Table 5.1. Comparison of Storage Engine Features

\begin{tabular}{|l|l|l|l|l|l|l|l|l|}
\hline Engine & $\begin{array}{l}\text { Multi- } \\
\text { Volume }\end{array}$ & $\begin{array}{l}\text { Mgnmt } \\
\text { Tools }\end{array}$ & $\begin{array}{l}\text { Cmmty, } \\
\text { Cmmrcl } \\
\text { Support }\end{array}$ & $\begin{array}{l}\text { Online } \\
\text { Backups }\end{array}$ & Shadowing & $\begin{array}{l}\text { Store } \\
\text { Access }\end{array}$ & $\begin{array}{l}\text { View } \\
\text { Access }\end{array}$ & ACID \\
\hline $\begin{array}{l}\text { MySQL } \\
\text { /MyISAM }\end{array}$ & $?$ & Yes & Yes & Yes & Yes & Yes & $?$ & No \\
\hline $\begin{array}{l}\text { MySQL } \\
\text { InnoDB }\end{array}$ & Yes & Yes & Yes & Yes & Yes & Yes & $?$ & Yes \\
\hline PostGres & & Yes & Yes & Yes & Yes & Yes & $?$ & Yes \\
\hline AllegroGraph & No & Few & Small & No(?) & No & No & No & Yes \\
\hline Sesame & No & Some & Yes & No (?) & No & No & No & $\begin{array}{l}\text { Yes } \\
(?)\end{array}$ \\
\hline Mulgara & No & No & Small & No(?) & No (?) & No & No & $\begin{array}{l}\text { Yes } \\
(?)\end{array}$ \\
\hline RDF Gateway & $?$ & Some & Small & $?$ & No(?) & Yes (?) & Yes (?) & $\begin{array}{l}\text { Yes } \\
(?)\end{array}$ \\
\hline BigOWLIM & No & Some & Yes & No (?) & No & No & No & Yes \\
\hline $\begin{array}{l}\text { OpenLink } \\
\text { Virtuoso }\end{array}$ & $?$ & Yes & $?$ & Yes & Yes & Yes & $?$ & Yes \\
\hline
\end{tabular}

Note that Oracle and SQL Server are not included in Table 5.1. It is assumed that, at the least, they support at least the features supported by MySQL and Postgres. 


\subsection{Server/API Software features}

Table 5.2. Server and API feature comparison

\begin{tabular}{|l|l|l|l|l|l|l|}
\hline System & Creation & Query support & Transitivity & Reification & $\begin{array}{l}\text { Community } \\
\text { Support }\end{array}$ & Reasoning \\
\hline Joseki (Jena) & Yes & Sparql & No & $\begin{array}{l}\text { Yes } \\
\text { (through } \\
\text { Jena }\end{array}$ & Yes & OWL-DL \\
\hline Sesame & Yes & SerQL & No & $?$ & Yes & RDFS \\
\hline Mulgara & Yes & Itql & No & $?$ & Small & Owl-Lite \\
\hline 3Store & $?$ & Sparql & $?$ & $?$ & Small & $?$ \\
\hline RDF Gateway & Yes & Proprietary & $\begin{array}{l}\text { RDFS } \\
\text { Reasoning }\end{array}$ & $?$ & Small & $\begin{array}{l}\text { RDFS } \\
\text { (Some } \\
\text { OWL) }\end{array}$ \\
\hline $\begin{array}{l}\text { OpenLink } \\
\text { Virtuoso }\end{array}$ & Yes & $\begin{array}{l}\text { Sparql, } \\
\text { Proprietary }\end{array}$ & No & Yes & Commercial & \begin{tabular}{l} 
RDFS \\
\hline AllegroGraph
\end{tabular} \\
Yes & $\begin{array}{l}\text { Sparql, } \\
\text { Prolog }\end{array}$ & Yes (Prolog) & Yes & Small & $\begin{array}{l}\text { Useful } \\
\text { subset of } \\
\text { OWL }\end{array}$ \\
\hline
\end{tabular}

\subsection{API/Backend Compatibility}

Table does not include rows for systems that are the only users of their RDF store (i.e., AllegroGraph).

Table 5.3. Compatability between backends and APIs

\begin{tabular}{|l|l|l|l|l|l|l|}
\hline System/Backend & MySql & Postgres & Oracle & $\begin{array}{l}\text { Sql } \\
\text { Server }\end{array}$ & Sesame & Mugara \\
\hline Joseki (Jena) & $\mathrm{Y}$ & $\mathrm{Y}$ & $\mathrm{Y}$ & $\mathrm{Y}$ & $\mathrm{Y}$ & $\mathrm{Y}$ \\
\hline Sesame & $\mathrm{N}(1)$ & $\mathrm{N}$ & $\mathrm{N}$ & $\mathrm{N}$ & $\mathrm{Y}$ & $\mathrm{N}$ \\
\hline Mulgara & $\mathrm{N}$ & $\mathrm{N}$ & $\mathrm{N}$ & $\mathrm{N}$ & $\mathrm{N}$ & $\mathrm{Y}$ \\
\hline 3Store & $\mathrm{Y}$ & $\mathrm{N}$ & $\mathrm{N}$ & $\mathrm{N}$ & $\mathrm{N}$ & $\mathrm{N}$ \\
\hline
\end{tabular}

Notes

1) Was compatible in version 1 , but not yet in version 2 


\subsection{Query Language Comparison}

Table 5.4. Query Language Comparison

\begin{tabular}{|l|l|l|l|l|}
\hline Language & Updates? & $\begin{array}{l}\text { Community } \\
\text { Support? }\end{array}$ & $\begin{array}{l}\text { Standards } \\
\text { Compliant? }\end{array}$ & Transitivity? \\
\hline Sparql & No & Yes & Yes & No \\
\hline SerQL (Sesame) & No & Yes & No & No \\
\hline Itql & Yes & Small & No & No(?) \\
\hline Prolog & No & No & No & Yes \\
\hline
\end{tabular}

\subsection{Performance Benchmarks}

\subsubsection{Data loading \& Provenance insertion}

Data loading and provenance insertion are evaluated by loading small RDF files, each representing a provenance record consisting of 5 triples, into the knowledge base. The amount of time it takes to load 1000 such records is compared against the current size of the knowledge base as an indication of system scalability. The results are graphed in Figure 5.1,

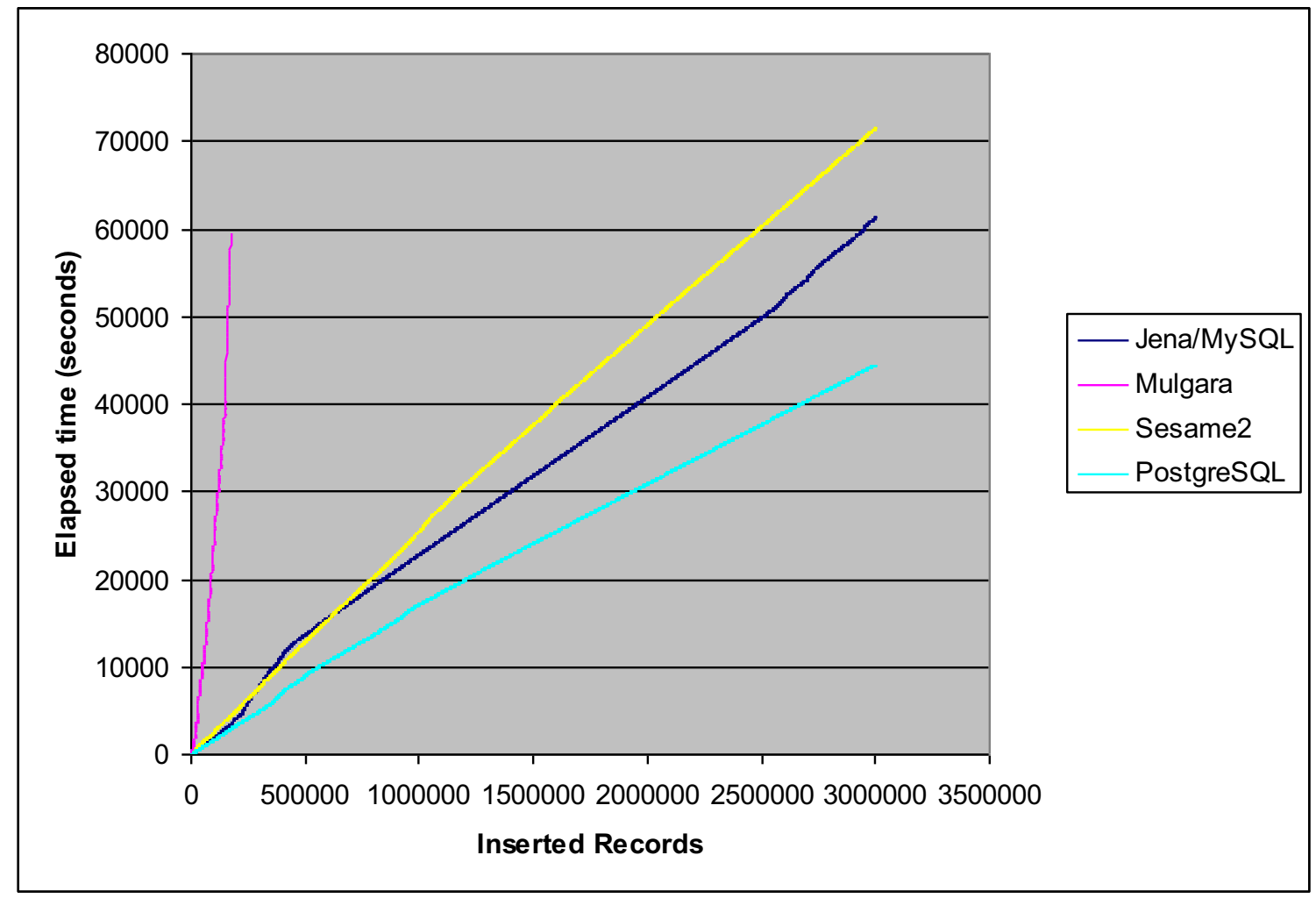

Figure 5.1. Data loading and Capacity

\subsubsection{Notes}

Jena with PostreSQL exhibits the best performance. Jena with MySQL exhibits scaleable insertion behavior. SesameV2's behavior is also scaleable. 
The Mulgara benchmark application initially aborted with an out-of-memory error after inserting 20000 records. Increasing memory for the server allowed more insertions to be made, but it still aborted after 174000 records.

AllegroGraph's documentation is very spotty on issues like backups and database parameters. I had problems setting a parameter called 'chunk size'. Setting it too small causes one kind of error, too big another kind. How to select a size is not specified, but it depends, I guess, on how many triples you plan to store. I was unable to determine a value that worked for the rdf file addition task - the server aborted if the number was too large, and created too many files if it was too small.

\subsubsection{Loading and querying LUBM data}

Different conclusions are drawn when the size of the rdf dataset is increased. Tests using the Lehigh University Benchmark (LUBM) (Guo, Pan et al. 2005). The LUBM

....is developed to facilitate the evaluation of Semantic Web repositories in a standard and systematic way. The benchmark is intended to evaluate the performance of those repositories with respect to extensional queries over a large data set that commits to a single realistic ontology. It consists of a university domain ontology, customizable and repeatable synthetic data, a set of test queries, and several performance metrics.(Semantic Web and Agent Technologies Lab 2007).

Table 5.5. Load times for LUBM data

\begin{tabular}{|l|l|l|}
\hline Dataset & Sesame2 Load time (Seconds) & Jena Load Time (seconds) \\
\hline 1 & 22,484 & 37,220 \\
\hline 2 & 27,269 & 47,077 \\
\hline 3 & 26,098 & 56,934 \\
\hline
\end{tabular}

A second benchmark used LUBM datasets to compare Jena and Sesame2 in load times and query performance. Three different LUBM datasets, each with approximately 6 million triples, were loaded into Jena and Sesame2 backends. The Jena system used MySql as a backend, Sesame used it's native file store. The results, shown in Table 5.5, indicate that while Jena is slower than Sesame2, the difference is not appreciably different for the size of datasets considered. The average time for Sesame to add 6 triples to a dataset was 13 microseconds, the average time for Jena was 31 microseconds.

The query results, summarized in Table 5.6, however, indicate that there are serious problems with Jena's query engine in some cases. 
Table 5.6. Results for queries

\begin{tabular}{|r|l|r|r|}
\hline & Query & Sesame 2 (ms) & Jena (ms) \\
\hline 1 & ?subj <named predicate> ?obj & 235 & 395761 \\
\hline 2 & <named subject> \$pred \$obj & 204 & 812 \\
\hline 3 & \$sub \$pred < named object> & 188 & 860 \\
\hline 4 & $\begin{array}{l}\text { \$sub < named predicate> < named object>. } \\
\text { \$sub \$pred \$obj }\end{array}$ & 203 & 750 \\
\hline 5 & \$sub \$pred \$obj FILTER(\$obj='Literal') & 187 & error: Out-of-memory \\
\hline 6 & \$sub \$pred 'Literal' & 188 & 593 \\
& & & error: no results \\
\hline 7 & \$sub \$pred \$obj FILTER regex(\$obj, 'Literal.') & 187 & error: Out-of-memory \\
\hline
\end{tabular}




\section{Conclusions}

\subsection{Backend Selection}

In the near future, we are still working with prototypes and data, and data integrity is not a serious issue. The large scale LUBM benchmarks show that the Sesame2 native store's performance is orders of magnitude better than the current database backends in query performance, so it will be used. Perhaps Sesame2 will support a different backend by the time we need it.

In the long term, a backend that uses a standard industry database, such as MySQL, Postgres, or Oracle is desired. Systems using native backends do not have the history that gives our team confidence in they're ability to provide database management tools, access control, $24 \mathrm{X} 7$ access, online backups, etc. Jena has recently provided an additional backend which can use commercial backends and is optimized for use on SPARQL Queries which may fit the bill (SDB 2007). In the long term, using Oracle as the backend is desired, since it is forseen that many customers will have experience with supporting Oracle. MySQL will be considered because its open source, it is installed, and the team is familiar with it.

\subsection{API Selection}

Two APIs have strong community support and meet the requirements of the team: Jena and Sesame1. Both can use MySql as a backend and both have similar strengths in supporting queries and in manipulating RDF graphs. The other APIs seem are either only available commercially, have limited community support, or are tied to proprietary backends.

Jena's strengths are its support for a wide variety of backends, strong community support, and support for complete OWL-DL reasoning. Drawbacks include a perception of over-complexity of the API and weaknesses in the query optimizer (logically equivalent queries can result in different execution times).

Sesame's strengths include strong community support, reported faster access speed, and previous usage at PNL. Its main drawback is lack of support for an RDBMS backend for the current release—-this makes direct performance comparisons difficult.

Given these difficulties and the functional similarity between the two APIs, both APIs will be supported for the nonce. 


\section{References}

Groth, P., S. Miles, et al. (2006). The Open Provenance Specification, The PROVENANCE Consortium.

Guo, Y., Z. Pan, et al. (2005). "LUBM: A Benchmark for OWL Knowledge Base Systems." Journal of Web Semantics 3(2): 158-182.

Lee, R. (2004). Scalability Report on Triple Store Applications. Cambridge, Mass., MIT.

Munroe, S., P. Groth, et al. (2006). Overview of the Provenance Specification Effort. The Open Provenance Specification, The PROVENANCE Consortium.

Portwin, K. and P. Parvatikar (2006). Scaling Jena in a commerical environment: The Ingenta MetaStore Project. Jena User Conference, Bristol, UK.

SDB(2007). "SDB - A SPARQL Database for Jena". http://jena.sourceforge.net/SDB/

Semantic Web and Agent Technologies Lab(2007). "LUBM Homepage". http://swat.cse.lehigh.edu/projects/lubm/index.htm

Triple Space Communication (2006). Semantic-based Knowledge and Content Systems, Triple Space Communication. 